\title{
The Multiplicative Spectrum and the Uniqueness of the Complete Norm Topology
}

\author{
J. C. $\operatorname{Marcos}^{\mathrm{a}}$, M. V. Velasco ${ }^{\mathrm{b}}$ \\ I.E.S. Generalife, C/ Huerta del Rasillo, 1, 18004- Granada (Spain) \\ ${ }^{b}$ Facultad de Ciencias, Universidad de Granada, 18071-Granada (Spain)
}

\begin{abstract}
.
We define the spectrum of an element $a$ in a non-associative algebra $A$ according to a classical notion of invertibility ( $a$ is invertible if the multiplication operators $L_{a}$ and $R_{a}$ are bijective). Around this notion of spectrum, we develop a basic theoretical support for a non-associative spectral theory. Thus we prove some classical theorems of automatic continuity free of the requirement of associativity. In particular, we show the uniqueness of the complete norm topology of $m$-semisimple algebras, obtaining as a corollary of this result a well-known theorem of Barry E. Johnson (1967).

The celebrated result of C.E. Rickart (1960) about the continuity of dense-range homomorphisms is also studied in the non-associative framework.

Finally, because non-associative algebras are very suitable models in genetics, we provide here a hint of how to apply this approach in that context, by showing that every homomorphism from a complete normed algebra onto a particular type of evolution algebra is automatically continuous.
\end{abstract}

\section{Introduction}

Many non-associative algebras have been used to model a great variety of phenomena in different scientific contexts of biology, physics, or engineering (see for instance [4, 11, 16, 19, 21]). However, a spectral theory for non-associative algebras still has not been developed, beyond of particular theories addressed only to some specific types of algebras (such as the spectral theory of Jordan algebras). However, many of the non-associative algebras that nowadays have great scientific interest do not belong to any of the well-known classes of non-associative algebras (such as Jordan, Lie or alternative algebras, among many others).

For instance, genetic algebras are natural examples of non-associative complete normed algebras arising in genetics. These algebras are not even power-associative, in general, and nevertheless they have strong connections with dynamical systems, graph theory, Markov processes, knot theory, and other topics. Consequently, apart from their mathematical interest, it turns out that its applications in physics and biology are remarkable. As was said in [26]:

2010 Mathematics Subject Classification. Primary 46H40, 46H70, 17D92

Keywords. Spectrum, spectral radius, non-associative complete normed algebra, radical, homomorphism, continuity, genetic algebra

Received: 18 April 2012; Accepted: 25 October 2013

Communicated by Pietro Aiena

Research supported by Junta de Andalucía grant FQM 0199

Email addresses: jucarmarsa@gmail.com (J. C. Marcos), vvelasco@ugr.es (M. V. Velasco) 
General genetic algebras are the product of interaction between biology and mathematics. Mendelian genetics introduced a new subject to mathematics: general genetic algebras. The study of these algebras reveals algebraic structures of Mendelian genetics, which always simplifies and shortens the way to understand genetic and evolutionary phenomena. Indeed, it is the interplay between purely mathematical structures and the corresponding genetic properties that makes this area so fascinating [...].

During the early days in this area, it appeared that the general genetic algebras or broadly defined genetic algebras, could be developed into a field of independent mathematical interest, because these algebras are in general not associative and do not belong to any of the well-known classes of nonassociative algebras such as Lie algebras, alternative algebras, or Jordan algebras. They possess some distinguishing properties that lead to many interesting mathematical results.

Throughout this paper, an algebra is a linear space $A$ over the field $\mathbb{K}$ (where either $\mathbb{K}=\mathbb{R}$ or $\mathbb{C}$ ) provided with a bilinear map $(a, b) \rightarrow a b$, from $A \times A \rightarrow A$. This bilinear map is named the multiplication of $A$.

If $(a b) c=a(b c)$ for every $a, b, c \in A$, then $A$ is associative. Similarly, if $a b=b a$ for every $a, b \in A$, then $A$ is commutative. Thus, associative algebras are particular examples of (non-associative) algebras.

We say that $A$ has a unit if there exists an element $e \in A$ such that $a e=e a=a$ for every $a \in A$. Obviously, the unit of $A$ is unique whenever it exists.

The notion of spectrum of an element in a Banach algebra (that is an associative complete normed algebra) is very satisfactory because, around it, properties of different nature (algebraic and topological) merge to give rise to a powerful spectral theory. Such theory becomes into the theoretical mathematical substrate over which some disciplines are developed (the Quantum Mechanics, for instance). We recall that for an associative algebra $A$ with a unit $e$, an element $a \in A$ is invertible if there exist $a^{-1} \in A$ such that $a a^{-1}=a^{-1} a=e$. Moreover, if $A$ is an associative complex algebra with a unit, then the spectrum of $a \in A$ is given by

$$
\sigma^{A}(a):=\{\lambda \in \mathbb{C}: a-\lambda e \text { is not invertible in } A\} .
$$

If $A$ is a complex associative algebra without a unit, then the spectrum of $a \in A$ is defined as $\sigma^{A_{1}}(a)$, where $A_{1}$ denotes the unitization of $A$. Similarly, if $A$ is a real algebra then, $\sigma^{A}(a):=\sigma^{A_{\mathbb{C}}}(a)$ where $A_{\mathbb{C}}$ is the complexification of $A$ (see [5] for details).

As said before, a non-associative spectral theory for general algebras is still in a preliminary state (see [27] and [17] for an approach). The main obstacle to be faced seems to be that, for these algebras, there is no notion of invertibility with so many good properties such as those in the associative setting. This is not surprising because associativity is a very strong property that gives rise to many consequences. For instance, it happens that if $A$ is a non associative algebra with a unit, $e$, and if $a \in A$ is such that $a b=b a=e$, for some $b \in A$ then, the uniqueness of $b$ is not guaranteed.

Nevertheless, since the beginning of the last century, (non-associative) division algebras have been considered in order to generalize the Gelfand-Mazur theorem, among other goals [3, 24]. In these works, a division algebra is defined as an algebra $A$ (possibly non-associative) such that for every non-zero element $a \in A$, left and right multiplication operators $L_{a}$ and $R_{a}$ are bijective on $A$. Motivated by this fact, in Definition 2.1, we define the $\mathbf{m}$-spectrum of an element $a$ in a complex algebra with a unit, $A$, as the set

$$
\sigma_{m}^{A}(a):=\left\{\lambda \in \mathbb{C}: L_{a}-\lambda I \text { or } R_{a}-\lambda I \text { is not bijective }\right\} .
$$

The spectrum of an element in either a complex algebra with a unit or a real algebra is defined in accordance with the associative case. As we show in Section 2, if $A$ is associative then $\sigma_{m}^{A}(a)=\sigma^{A}(a)$, for every $a \in A$, and consequently, the m-spectrum is nothing but a generalization of the classical spectrum to the framework 
of (possibly non-associative) algebras. The aim of this paper is to develop a theoretical basis for a nonassociative spectral theory around the concept of the m-spectrum.

In Section 2 we give some basic properties of the m-spectrum of an element. Indeed, the $\mathbf{m}$-spectral radius of $a \in A$ is defined as the minimum radius $\rho(a)$ of a closed disc centered in the origin containing the set $\sigma_{m}^{A}(a)$, and, in Proposition 2.7, it is characterized in terms of the norm.

In Sections 3 and 4, some classical automatic continuity theorems for homomorphisms are extended to the non-associative setting. Actually, surjective homomorphisms are studied in Section 3, while Section 4 is addressed to homomorphisms that need not be surjective. Note that a homomorphism $\theta: A \rightarrow B$ between two complete normed algebras $A$ and $B$ can be regarded as a dense-range homomorphism $\theta: A \rightarrow \overline{\theta(A)}$, where $\overline{\theta(A)}$ denotes the closure of $\theta(A)$ in $B$. Therefore the epimorphisms are the target of Section 3 while the dense-range homomorphisms are that of Section 4.

In Definition 3.1 we state that an algebra $A$ is multiplicatively semisimple (or $\mathbf{m}$-semisimple for short) if there are no non-zero ideals contained in the set $\{a \in A: \rho(a)=0\}$ of elements of $A$ with zero m-spectral radius. If $A$ is associative this means that the Jacobson radical of $A$ is zero (see [20, Theorem 4.3.6]).

In Theorem 3.5, we prove that every surjective homomorphism from a complete normed algebra onto a msemisimple complete normed algebra, is continuous. Consequently, complete normed $m$-semisimple algebras have a unique complete norm topology (Corollary 3.6). Particularly, whenever $A$ is associative, we obtain a well known theorem of B. E. Johnson [14] (see also [5, 6, 20]) that in words of T. Palmer is a "cornerstone of the Banach algebra theory". Moreover, Theorem 3.5 and Corollary 3.6 improve the main results in [17]. Also, because determining the set of elements having $\mathrm{m}$-spectral radius equal to zero is not difficult (in the level of abstraction that we are dealing with) these results can be easily applied, as the Example 3.7 shows.

A main classical result about the automatic continuity of dense range homomorphisms, is a theorem of C. E. Rickart [22] which asserts that if $B$ is strongly semisimple then every dense range-homomorphism $\theta: A \rightarrow B$ is continuous. This result makes sense for non-associative algebras (by defining the strongly-semisimple algebras as those such that the intersection of its two-sided maximal modular ideals is zero). In Proposition 4.1, we show that to determine whenever the associativity is superfluous in Rickart's theorem, it is not restrictive to consider that $A$ and $B$ are complete normed algebras with a unit, and that $B$ is simple. In this setting, Theorem 4.6 provides a sufficient condition in the range of $\theta$ that implies the continuity of $\theta$, namely that $\theta(A)$ is spectrally admissible in the sense of Definition 4.3. Since dense subalgebras of (associative) Banach algebras with a unit are always spectrally admissible, we obtain the classical theorem of Rickart as a corollary of Theorem 4.6 .

Additionally, in Proposition 4.7, we show that if $A$ and $B$ are complete normed algebras and if $\theta: A \rightarrow B$ is an homomorphism such that $\theta(A)$ contains some element having empty surjective m-spectrum, then $\theta$ is discontinuous. This pathology is showed in Example 4.8, where there is exhibited a complete normed algebra, $B$, simple with a unit, containing a dense subalgebra, $B_{0}$, which has elements with empty surjective spectrum. Therefore, if $A$ is a complete normed algebra and if $\theta: A \rightarrow B$ is an homomorphism such that $\theta(A)=B_{0}$ then $\theta$ is a discontinuous dense-range homomorphism.

As we have seen, the nature of this paper is theoretical. Nevertheless, in Section 5, we give an idea of how to obtain applications of some of these results in the framework of evolution algebras (very relevant algebras in non-Mendelian Genetics, and hence in Molecular Biology). For a wider study addressed specifically to the spectral theory of evolution algebras, see [18]. 


\section{The m-spectrum}

As said already, from now on, by an algebra we mean a linear space $A$ over the field $\mathbb{K}$ (where either $\mathbb{K}=\mathbb{R}$ or $\mathbb{K}=\mathbb{C}$ ) provided with a bilinear map $(a, b) \rightarrow a b$, from $A \times A \rightarrow A$.

An algebra $A$ without a unit can be unitized by defining the algebra $A_{1}=A \oplus \mathbb{K} 1$, where $\mathbb{K}$ denotes the base field of $A$, provided with the product

$$
(a+\lambda \mathbf{1})(b+\mu \mathbf{1})=(a b+\mu a+\lambda b)+\lambda \mu \mathbf{1},
$$

for every $a, b \in A$ and $\lambda, \mu \in \mathbb{K}$. Thus, we obtain an algebra $A_{1}$ that has a unit, 1, and contains $A$ as an ideal.

Similarly, a real algebra $A$ can be complexified by defining the complex algebra $A_{\mathbb{C}}=A \oplus i A$ where $(r+i s) a=r a+i s a$, for every $a \in A$ and $r, s \in \mathbb{R}$, and $(a+i b)(c+i d)=a c-b d+i(a d+b c)$, for every $a, b \in A$. As in the associative case it is easy to check that, if $A$ is a real algebra without a unit, then $\left(A_{\mathbb{C}}\right)_{1}=\left(A_{1}\right)_{\mathbb{C}}$ (see [5, Section 1.13] for details, by observing that the associativity is not relevant for this particular topic).

We recall that if $A$ is an algebra and if $a \in A$, then left multiplication operator associated to $a$ is defined as the operator $L_{a}: A \rightarrow A$ given by $L_{a}(b)=a b$, for every $b \in A$. Similarly, the right multiplication operator associated to $a \in A$ is the operator $R_{a}: A \rightarrow A$ given by $R_{a}(b)=b a$, for every $b \in A$.

Definition 2.1. Let $A$ be a complex algebra with a unit. We say that $a \in A$ is multiplicatively invertible (or $m$-invertible) whenever both $L_{a}$ and $R_{a}$ are bijective.

We define the multiplicative spectrum (or the $m$-spectrum) of $a \in A$ as the set

$$
\sigma_{m}^{A}(a):=\left\{\lambda \in \mathbb{C}: L_{a}-\lambda I \text { or } R_{a}-\lambda I \text { is not bijective }\right\} .
$$

If $A$ is a complex algebra without a unit then we define the $m$-spectrum of $a \in A$ as the set $\sigma_{m}^{A_{1}}(a)$, where $A_{1}$ denotes the unitization of $A$. of $A$.

Finally, if $A$ is a real algebra then we define the $m$-spectrum of $a \in A$ as $\sigma_{m}^{A_{\mathrm{C}}}(a)$, where $A_{\mathbb{C}}$ is the complexification

Let $B$ be an associative complex algebra with a unit, $e$. Note that if $b \in B$ is invertible (which means that there exists $b^{-1} \in B$ such that $b b^{-1}=b^{-1} b=e$ ) then, $L_{b}$ and $R_{b}$ are bijective. Indeed $L_{b}^{-1}=L_{b^{-1}}$ and $R_{b}^{-1}=R_{b^{-1}}$. Conversely, if $L_{b}$ and $R_{b}$ are bijective then, from the associativity of $B$, we have $L_{b}^{-1}(e)=R_{b}^{-1}(e)=b^{-1}$. The spectrum of $b$ in $B$ is given by $\sigma^{B}(b):=\{\lambda \in \mathbb{C}: b-\lambda e$ is not invertible in $B\}$, and therefore,

$$
\sigma^{B}(b)=\sigma_{m}^{B}(b), \quad(b \in B) .
$$

Thus the m-spectrum is an extension of the classical notion of spectrum of an element in an associative algebra to the non-associative setting, as noted already.

Let $A$ be a complex algebra with a unit and let $\mathcal{L}(A)$ denote the algebra of all linear operators $T: A \rightarrow A$. Since $\mathcal{L}(A)$ is an associative algebra with a unit, $I$, even when $A$ is not associative, we have that the spectrum of $T \in \mathcal{L}(A)$ is given by

$$
\sigma^{\mathcal{L}(A)}(T)=\{\lambda \in \mathbb{C}: T-\lambda I \text { is not bijective }\} .
$$

Consequently, for every $a \in A$, we have

$$
\sigma_{m}^{A}(a):=\sigma^{\mathcal{L}(A)}\left(L_{a}\right) \cup \sigma^{\mathcal{L}(A)}\left(R_{a}\right) .
$$

Suppose now that $A$ is a complex algebra without a unit. Then

$$
\sigma_{m}^{A}(a):=\sigma_{m}^{A_{1}}(a)=\sigma^{\mathcal{L}\left(A_{1}\right)}\left(L_{a}\right) \cup \sigma^{\mathcal{L}\left(A_{1}\right)}\left(R_{a}\right) .
$$

In the next result we formulate the m-spectrum of an element $a$ in an algebra $A$ without a unit in terms of $A$ (that is without explicit mention of $A_{1}$ ). 
Proposition 2.2. If $A$ is an algebra without a unit, and if $A_{1}$ is its unitization, then

$$
\begin{aligned}
\sigma^{\mathcal{L}(A)}\left(L_{a}\right) \backslash\{0\} & =\sigma^{\mathcal{L}\left(A_{1}\right)}\left(L_{a}\right) \backslash\{0\}, \\
\sigma^{\mathcal{L}(A)}\left(R_{a}\right) \backslash\{0\} & =\sigma^{\mathcal{L}\left(A_{1}\right)}\left(R_{a}\right) \backslash\{0\},
\end{aligned}
$$

for every $a \in A$. Consequently, $\sigma_{m}^{A}(a)=\sigma^{\mathcal{L}(A)}\left(L_{a}\right) \cup \sigma^{\mathcal{L}(A)}\left(R_{a}\right) \cup\{0\}$.

Proof. We suppose that $A$ is a complex algebra because it is not restrictive to do this.

Let $a \in A$ and $\lambda \in \mathbb{C} \backslash\{0\}$. It is straightforward to check that $L_{a}-\lambda I: A \rightarrow A$ is injective if and only if $L_{a}-\lambda I: A_{1} \rightarrow A_{1}$ is injective. Similarly, if the map $L_{a}-\lambda I: A \rightarrow A$ is surjective, then there exists $b \in A$ such that $\left(L_{a}-\lambda I\right)(b)=\frac{1}{\lambda} a$. Therefore $\left(L_{a}-\lambda I\right)\left(b-\frac{1}{\lambda} \mathbf{1}\right)=\mathbf{1}$, and the surjectivity of $L_{a}-\lambda I: A_{1} \rightarrow A_{1}$ follows.

Conversely, from the surjectivity of $L_{a}-\lambda I: A_{1} \rightarrow A_{1}$, the surjectivity of $L_{a}-\lambda I: A \rightarrow A$ is obtained directly. Consequently, $\sigma^{\mathcal{L}\left(A_{1}\right)}\left(L_{a}\right) \backslash\{0\}=\sigma^{\mathcal{L}(A)}\left(L_{a}\right) \backslash\{0\}$.

Similarly $\sigma^{\mathcal{L}\left(A_{1}\right)}\left(R_{a}\right) \backslash\{0\}=\sigma^{\mathcal{L}(A)}\left(R_{a}\right) \backslash\{0\}$. Moreover, by $(1)$, we have $\sigma_{m}^{A}(a):=\sigma_{m}^{A_{1}}(a)=\sigma^{\mathcal{L}\left(A_{1}\right)}\left(L_{a}\right) \cup \sigma^{\mathcal{L}\left(A_{1}\right)}\left(R_{a}\right)$. Also 0 is in $\sigma^{\mathcal{L}\left(A_{1}\right)}\left(L_{a}\right) \cap \sigma^{\mathcal{L}\left(A_{1}\right)}\left(R_{a}\right)$ because the maps $L_{a}, R_{a}: A_{1} \rightarrow A_{1}$ are not surjective. Thus, we conclude that $\sigma_{m}^{A}(a)=\sigma^{\mathcal{L}(A)}\left(L_{a}\right) \cup \sigma^{\mathcal{L}(A)}\left(R_{a}\right) \cup\{0\}$.

A normed algebra is an algebra $A$ with a norm $\|\cdot\|$ such that $\|a b\| \leq\|a\|\|b\|$ for every $a, b \in A$ (that is, up to an equivalent renorming, a norm making the product of $A$ continuous).

If $A$ is a normed algebra, then $L_{a}$ and $R_{a}$ belong to $L(A)$, the associative algebra of all bounded linear operators on $A$. Moreover, if $A$ is complete then by the Banach isomorphism theorem, if $L_{a}$ is bijective, then $L_{a}^{-1}$ is continuous. This means that $L_{a}$ is invertible in $\mathcal{L}(A)$ if and only if $L_{a}$ is invertible in $L(A)$, and therefore,

$$
\sigma^{\mathcal{L}(A)}\left(L_{a}\right)=\sigma^{L(A)}\left(L_{a}\right) .
$$

Similarly $\sigma^{\mathcal{L}(A)}\left(R_{a}\right)=\sigma^{L(A)}\left(R_{a}\right)$. Thus, for every element $a$ in a complete normed algebra $A$ we have that $\sigma_{m}^{A}(a)=\sigma^{L(A)}\left(L_{a}\right) \cup \sigma^{L(A)}\left(R_{a}\right)$ if $A$ has a unit, while $\sigma_{m}^{A}(a)=\sigma^{L(A)}\left(L_{a}\right) \cup \sigma^{L(A)}\left(R_{a}\right) \cup\{0\}$ otherwise. Consequently we obtain the next result.

Corollary 2.3. If $A$ is a complete normed algebra, and if $a \in A$ then $\sigma_{m}^{A}(a) \backslash\{0\} \subseteq \sigma^{L(A)}\left(L_{a}\right) \cup \sigma^{L(A)}\left(R_{a}\right)$.

Now it is very easy to prove the following fact.

Proposition 2.4. Let $A$ be a complete normed algebra. Then the set of m-invertible elements of $A$ is open in $A$.

Proof. If $a \in A$ is m-invertible then $L_{a}$ and $R_{a}$ are invertible elements in the Banach algebra $L(A)$ so that, by [5, Theorem 1.2.11], the open ball $B_{L(A)}\left(L_{a},\left\|L_{a}^{-1}\right\|^{-1}\right)$ as well as $B_{L(A)}\left(R_{a},\left\|R_{a}^{-1}\right\|^{-1}\right)$ are contained in the set of all invertible elements in $L(A)$. Consequently, if $0<r \leq \min \left\{\left\|L_{a}^{-1}\right\|^{-1},\left\|R_{a}^{-1}\right\|^{-1}\right\}$, and if $b \in B_{A}(a, r)$ then, the operators $L_{b}$ and $R_{b}$ are invertible, so that $b$ is $\mathrm{m}$-invertible in $A$. This proves that the set of $\mathrm{m}$-invertible elements in $A$ is open in $A$.

From [5, Theorem 1.5.8] and Corollary 2.3 the next result follows.

Proposition 2.5. If $A$ is a complete normed algebra and if $a \in A$ then, $\sigma_{m}^{A}(a)$ is a non-empty compact subset of $\mathbb{C}$. Moreover $|\lambda| \leq\|a\|$, for every $\lambda \in \sigma_{m}^{A}(a)$.

According to [20], an associative normed algebra $B$ is spectral if $\sigma^{B}(b) \subseteq \mathbb{D}_{\|b\|,}$ where $\mathbb{D}_{\|b\|}:=\{\lambda \in \mathbb{C}:|\lambda| \leq\|b\|\}$. Therefore, the above result shows the spectral behavior of complete normed (non-associative) algebras. 
Definition 2.6. Let $A$ be an algebra. The $m$-spectral radius of $a \in A$ is $\rho(a):=\sup \left\{|\lambda|: \lambda \in \sigma_{m}^{A}(a)\right\}$ whenever $\sigma_{m}^{A}(a)$ is non-empty, and $\rho(a)=0$ otherwise. Thus $0 \leq \rho(a) \leq \infty$.

The next result is a kind of Beurling-Gelfand formula for the m-spectrum.

Proposition 2.7. Let $A$ be a complete normed algebra, and let $a \in A$. Then $\rho(a)=\max \left\{\lim _{n \rightarrow \infty}\left\|L_{a}^{n}\right\|^{\frac{1}{n}}, \lim _{n \rightarrow \infty}\left\|R_{a}^{n}\right\|^{\frac{1}{n}}\right\}$.

Proof. By the Beurling-Gelfand formula (see [5, Theorem 1.5.8] or [6]) we have the equalities

$$
\begin{aligned}
& \max \left\{|\lambda|: \lambda \in \sigma^{L(A)}\left(L_{a}\right)\right\}=\lim _{n \rightarrow \infty}\left\|L_{a}^{n}\right\|^{\frac{1}{n}}, \\
& \max \left\{|\lambda|: \lambda \in \sigma^{L(A)}\left(R_{a}\right)\right\}=\lim _{n \rightarrow \infty}\left\|R_{a}^{n}\right\|^{\frac{1}{n}} .
\end{aligned}
$$

Thus the result follows from Corollary 2.3.

\section{The m-semisimplicity and the uniqueness of the complete norm topology}

A classical result of Johnson [14] that, as noted before, "is a cornerstone of general Banach algebras theory" (quoting [20, p. 554]) establishes that a semisimple Banach algebra has a unique complete norm topology. We recall that an associative algebra $B$ is semisimple if its Jacobson radical is equal to zero.

The radical of Jacobson of an associative algebra can be characterized in terms of the spectrum. As said in [20, p. 189] (see also [20, Theorem 4.3.6]) the Jacobson radical [of an associative algebra B] can be described as the largest ideal on which the spectral radius of each element is identically 0 . Consequently $B$ is semisimple if its radical is zero, that is if $\{0\}$ is the only ideal contained in set of elements having spectral radius equal to zero.

Since Definition 2.6 provides us with a notion of spectral radius for non-associative algebras, the above fact suggests the next definition.

Definition 3.1. We say that an algebra $A$ is $m$-semisimple if $\{0\}$ is the only ideal of $A$ contained in $\{a \in A: \rho(a)=0\}$, the set of all elements in A having m-spectral radius equal to zero.

We recall that the Jacobson $\operatorname{radical}, \operatorname{Rad}(B)$, of an associative algebra, $B$, is also the intersection of the primitive ideals of $B$. By the usual convention, $\operatorname{Rad}(B)=B$ if there are no primitive ideals of $B$. In [17], the Jacobson radical $\operatorname{Rad}(A)$ of an algebra $A$ (possibly non associative) was defined as the intersection of all primitive ideals of $A$. There, the primitive ideals of $A$ were defined as the largest ideal contained in a one-sided maximal modular ideal of $A$. Moreover, semisimple algebras were defined as those algebras $A$ such that $\operatorname{Rad}(A)=\{0\}$. As a main result [17, Theorem 20], it was proved that a semisimple complete normed algebras has a unique complete norm topology. Therefore, associativity is a superfluous assumption in the aforementioned classical result of Johnson [14].

Our next goal is to prove that the class of m-semisimple algebras is wider than that of semisimple algebras but, nevertheless, m-semisimple complete normed algebras enjoy the property of the uniqueness of the complete norm topology (which improves [17, Theorem 20]).

An element $a$ in an algebra $A$ is quasi-invertible if $a$ has a left and a right quasi-inverse (that is, there exist $b, c \in A$ such that $a+b-a b=0=a+c-c a)$. In contrast to the associative case, $\operatorname{Rad}(A) \operatorname{may}$ contain elements that are not quasi-invertible. Nevertheless, in [17, Proposition 8], it was proved that if $Q$ is a quasi-invertible ideal then, $Q \subseteq \operatorname{Rad}(A)$. From this fact we will deduce the next result.

Proposition 3.2. Let $A$ be a semisimple complete normed algebra. Then $A$ is $m$-semisimple. 
Proof. Assume that $A$ is not m-semisimple. Then there exists a non-zero ideal of $A$, say $Q$, contained in $\{a \in A: \rho(a)=0\}$. Consequently, $1 \notin \sigma_{m}^{A}(a)$, for every $a \in Q$, which shows that both $\left(L_{a}-I\right)$ and $\left(R_{a}-I\right)$ are bijective and hence invertible in $L(A)$, so that there exists $T$ and $S$ in $L(A)$ such that $\left(L_{a}-I\right) T=I$ and $\left(R_{a}-I\right) S=I$. This shows that $T(a)$ is a left quasi-inverse of $a$ and $S(a)$ is a right quasi-inverse of $a$, so that $Q$ is a quasi-invertible ideal. By [17, Proposition 8], we conclude that $Q \subseteq \operatorname{Rad}(A)=\{0\}$, a contradiction.

Next we provide an example of a m-semisimple algebra which is not semisimple. Therefore the equivalence between the semisimplicity and the m-semisimplicity does not hold beyond the associative case.

Example 3.3. Consider the complex algebra A generated by $\{a, b, c\}$ whose multiplication table is given by

\begin{tabular}{|c||c|c|c|} 
& $a$ & $b$ & $c$ \\
\hline \hline$a$ & $a$ & 0 & 0 \\
\hline$b$ & $b$ & 0 & 0 \\
\hline$c$ & 0 & $a$ & $a$ \\
\hline
\end{tabular}

Given a subset $S \subset A$ let denote by $\langle S\rangle_{\text {left }}$ the left ideal of A generated by $S$, and similarly denote by $\langle S\rangle_{\text {right }}$ the corresponding right ideal. It turns out that $\langle a\rangle_{\text {left }}=\mathbb{C} a \oplus \mathbb{C} b$, because if $\alpha, \beta, \gamma \in \mathbb{C}$ then

$$
\begin{array}{r}
(\alpha a+\beta b+\gamma c) a=\alpha a+\beta b \\
(\alpha a+\beta b+\gamma c) b=\gamma a .
\end{array}
$$

Also, $\langle a\rangle_{\text {right }}=\mathbb{C} a$ because $a(\alpha a+\beta b+\gamma c)=\alpha a$. Consequently, the ideal generated by $\{a\}$ is $\langle a\rangle=\mathbb{C} a \oplus \mathbb{C} b$. In [17, Theorem 10] it was proved that $\operatorname{Rad}(A)$ is the largest ideal contained into the set

$$
\left\{w \in A:\langle A(1-w)\rangle_{\text {left }}=A=\langle(1-w) A\rangle_{\text {right }}\right\} .
$$

Since $c-c(\alpha a+\beta b)=c-\beta a, c(c-\beta a)=a$, and $b a=b$, we deduce that

$$
\langle A(1-(\alpha a+\beta b))\rangle_{\text {left }}=A .
$$

Similarly $b-(\alpha a+\beta b) b=b$, and $c-(\alpha a+\beta b) c=c$. Moreover $c^{2}=a$, and hence,

$$
\left\langle(1-(\alpha a+\beta b) A\rangle_{\text {right }}=A .\right.
$$

This proves that the ideal generated by $\{a\}$ is contained in the set given by (2), so that $a \in \operatorname{Rad}(A)$, and hence $A$ is not semisimple.

On the other hand, we claim that

$$
\mathbb{C} b \cup \mathbb{C} c=\{u \in A: \rho(u)=0\} .
$$

This fact shows that $A$ is $m$-semisimple since a belongs to both the ideal generated by $\{b\}$ and the one generated by $\{c\}$ (because $c b=a$ ). Consequently these ideals are not contained into $\mathbb{C} b \cup \mathbb{C} c$, and hence $\{0\}$ is the only ideal contained in the set $\{a \in A: \rho(a)=0\}$, as desired.

To prove the claim (3), note that if $\beta, \lambda \in \mathbb{C}$ with $\beta \neq 0$ then, the roots of $\lambda^{2}-\lambda-\beta \gamma=0$ are eigenvalues of $L_{a+\beta b+\gamma c}$ (in fact, if $\lambda^{2}-\lambda-\beta \gamma=0$ then $\left.\left(L_{a+\beta b+\gamma c}-\lambda I\right)\left(\frac{\lambda}{\beta} a+b\right)=0\right)$. Therefore,

$$
\sigma_{m}^{A}(a+\beta b+\gamma c) \neq\{0\} .
$$

Similarly, $\sigma_{m}^{A}(a+\gamma c) \neq\{0\}$ (because $\left.\left(L_{a+\gamma c}-I\right)(a)=0\right)$. On the other hand, if $\alpha \in \mathbb{C} \backslash\{0\}$, then

$$
\left(L_{b+\alpha c}+\sqrt{\alpha} I\right)(\sqrt{\alpha} a-b)=0,
$$

and hence $\sigma_{m}^{A}(b+\alpha c) \neq\{0\}$. Finally it is easy to check that $\sigma_{m}^{A}(b)=\sigma_{m}^{A}(c)=\{0\}$, because the operators $L_{b}-\lambda I, R_{b}-\lambda I$, $L_{c}-\lambda I$ and $R_{c}-\lambda I$ are bijective for every $\lambda \neq 0$, while $L_{b}$ and $R_{c}$ are not. This proves the claim (3), and hence $A$ is m-semisimple. 
We recall that if $X$ is a complex linear space then the surjective spectrum of a linear operator $T \in \mathcal{L}(X)$ is defined as the subset of $\sigma^{\mathcal{L}(X)}(T)$ given by

$$
\sigma_{s u}^{X}(T):=\{\lambda \in \mathbb{C}: T-\lambda I \text { is not surjective on } X\} .
$$

The following result is well known (see for instance [15, Proposition 1.3.2 (a)]).

Lemma 3.4. If $X$ is a Banach space and if $T \in L(X)$ then $\sigma_{s u}^{X}(T)$ is a non-empty closed set that contains the boundary of $\sigma^{L(X)}(T)$.

We also recall that $X$ and $Y$ are normed spaces and if $\theta: X \rightarrow Y$, is a linear map then, the separating subspace of $\theta$ is the closed subspace of $Y$ defined by

$$
\mathcal{S}(\theta):=\left\{y \in Y: \exists x_{n} \rightarrow 0 \text { in } X \text { such that } \theta\left(x_{n}\right) \rightarrow y\right\} .
$$

Moreover, if $X$ and $Y$ are Banach spaces then closed graph theorem asserts that $\theta$ is continuous precisely whenever $\mathcal{S}(\theta)=\{0\}$.

Theorem 3.5. Let $A$ and be complete normed algebras, such that $B$ is $m$-semisimple, and $\theta: A \rightarrow B$ a surjective homomorphism. Then $\theta$ is continuous.

Proof. In [17, Lemma 18] it was proved that if $A$ and $B$ are complete normed algebras and $\theta: A \rightarrow B$ is a homomorphism from $A$ onto $B$, then

$$
\sigma_{s u}^{B}\left(L_{b}\right)=\sigma_{s u}^{B}\left(R_{b}\right)=\{0\}
$$

for every $b \in \mathcal{S}(\theta)$. On the other hand, by Lemma 3.4, we have that $\sigma_{s u}^{B}\left(L_{b}\right)$ is closed and contains $\partial \sigma^{L(B)}\left(L_{b}\right)$ (the boundary of the spectrum of $L_{b}$ in $L(B)$ ). Therefore $\sigma^{L(B)}\left(L_{b}\right)=\{0\}$, and similarly $\sigma^{L(B)}\left(R_{b}\right)=\{0\}$. Thus $\sigma_{m}^{B}(b)=0$ for every $b \in \mathcal{S}(\theta)$, by Corollary 2.3 , and consequently,

$$
\mathcal{S}(\theta) \subseteq\{b \in B: \rho(b)=0\} .
$$

From the m-semisimplicity of $B$ we obtain $\mathcal{S}(\theta)=\{0\}$, and therefore by the closed graph theorem $\theta$ is continuous.

A complete normed algebra $A$ has a unique complete norm topology whenever any other complete algebra norm on $A$ is equivalent to the original algebra norm (so that both norms define the same topology). From the above theorem, applied to the homomorphism given by the identity on an m-semisimple algebra endowed with two complete norms, we obtain the next result.

Corollary 3.6. Complete normed m-semisimple algebras have a unique complete norm topology.

The above result improves [17, Corollary 21], and shows that associativity is a superfluous hypothesis in the aforementioned classical result of B. Johnson [14]. Moreover, in many cases, the above results is quite easy to apply, as we show next.

Example 3.7. Let $B$ be the algebra consisting in all elements $b=\sum_{n \geq 1} \beta_{n} e_{n}$, such that $\sum_{n=1}^{\infty}\left|\beta_{n}\right|<\infty$, provided with the multiplication table given by $e_{n} e_{m}=0$ if $n \neq m$, and $e_{n}^{2}=\frac{e_{n}+e_{n+1}}{2}$. Then $B$ is a complete normed algebra, with the norm given by $\|b\|=\sum_{n=1}^{\infty}\left|\beta_{n}\right|$. For $b=\sum_{n=1}^{\infty} \beta_{n} e_{n} \in B \backslash\{0\}$, let $k_{0}:=\min \left\{n \in \mathbb{N}: \beta_{k} \neq 0\right\}$. Then $\frac{\beta_{k_{0}}}{2} \in \sigma_{m}^{B}(b)$ because $e_{k_{0}}$ is not in the range of $L_{b}-\frac{\beta_{k_{0}}}{2} I$. This proves that $B$ is m-semisimple. Therefore every surjective homomorphism from a complete normed algebra onto B is automatically continuous. In particular, B has a unique complete norm topology. 


\section{The $\mathrm{m}$-spectrum and the continuity of dense-range homomorphisms.}

In this Section, we study the role of the m-spectrum concerning the automatic continuity of dense-range homomorphisms.

In the associative setting, a celebrated theorem by Rickart [22] states that if $A$ and $B$ are Banach algebras, if $B$ is strongly semisimple, and if $\theta: A \rightarrow B$ is a dense-range homomorphism, then $\theta$ is continuous. Since the strong radical of $A$ is defined as the intersection of all two-sided maximal modular ideals of $A$ it turns out that this notion does not involve explicitly the associative property, so that it can be extended directly to the setting of non-associative algebras. Thus it is natural to ask if associativity is a superfluous hypothesis in Rickart's theorem. A survey paper about this question is [25], and recent advances are described in [27]. Next, we show that the problem can be reduced to the case where the algebras $A$ and $B$ are unital and $B$ is simple.

Proposition 4.1. The following statements are equivalent:

(i) Every dense range homomorphism from a complete normed algebra into a strongly semisimple complete normed algebra is continuous.

(ii) If $A$ and $B$ are unital complete normed algebras and if $B$ is simple, then every dense range homomorphism $\theta: A \rightarrow B$ is continuous.

Proof. Assertion (i) follows from the fact that simple algebras are strongly semisimple.

Conversely, suppose that assertion (ii) is satisfied, and let $\theta: A \rightarrow B$ be a dense-range homomorphism from a complete normed algebra $A$ into a strongly semisimple complete normed algebra $B$. Let $M$ be a maximal modular ideal of $B$. From [27, Corollary 6] we obtain that $M$ is closed and hence $\widetilde{B}:=B / M$ is a simple complete normed algebra with a unit, so that, if $\pi: B \rightarrow B / M$ is the canonical projection then, $\widetilde{\theta}:=\pi \theta: A \rightarrow \widetilde{B}$ is a dense-range homomorphism. If $A$ has not a unit then, we can extend $\widetilde{\theta}$ to $A_{1}=A \oplus \mathbb{K} \mathbf{1}$, the unitization of $A$, by defining $\widetilde{\theta}_{1}: A_{1} \rightarrow B$ as $\widetilde{\theta}_{1}(a+\lambda \mathbf{1})=\widetilde{\theta}(a)+\lambda e$, for every $a \in A$, where $e$ denotes the unit of $\widetilde{B}$, and it follows that $\widetilde{\theta}$ is continuous precisely whenever $\widetilde{\theta}_{1}$ is continuous. Therefore by replacing $\widetilde{\theta}: A \rightarrow \widetilde{B}$ by $\widetilde{\theta}_{1}: A_{1} \rightarrow \widetilde{B}$ if needed, it is not restrictive to assume that $A$ has a unit, and hence $\widetilde{\theta}$ is unital. Thus, $\widetilde{\theta}:=\pi \theta: A \rightarrow \widetilde{B}$ is continuous by assertion (ii). Consequently, if $a_{n}$ is a sequence in $A$ such that $a_{n} \rightarrow 0$ then, $\widetilde{\theta}\left(a_{n}\right)=\pi \theta\left(a_{n}\right) \rightarrow \widetilde{\theta}(0)=M$, which proves that $\mathcal{S}(\theta)$, the separating subspace of $\theta$, is contained in $M$. Consequently $\mathcal{S}(\theta)$ is contained in the strong radical of $B$, and therefore $\mathcal{S}(\theta)=\{0\}$, which proves that $\theta$ is continuous.

The above result shows that the problem of determining whether the surjectivity is superfluous in Rickart's dense-range homomorphism theorem can be reformulated as follows:

Problem 4.2. Let $A$ and $B$ be complete normed algebras with a unit such that $B$ is simple, and let $\theta: A \rightarrow B$ be a dense range homomorphism. Is $\theta$ automatically continuous?

A property of the range of the homomorphism $\theta$ that implies the continuity of $\theta$ is given next. Recall that if $r>0$, then $\mathbb{D}_{r}:=\{\lambda \in \mathbb{C}:|\lambda| \leq r\}$.

Definition 4.3. Let $B$ be a normed algebra. We say that $B$ is spectrally admissible if there exists $M>0$ such that for every $b \in B$, we have that $\sigma_{s u}^{B}(b) \cap M \mathbb{D}_{\|b\|} \neq \emptyset$, where $\sigma_{s u}^{B}(b):=\sigma_{s u}^{B}\left(L_{b}\right) \cup \sigma_{s u}^{B}\left(R_{b}\right)$. Moreover, the set $\sigma_{s u}^{B}(b)$ is the surjective $m$-spectrum of $b \in B$.

A class of spectrally admissible algebras is that of the complete normed algebras because of their spectral nature, as we show next. 
Proposition 4.4. If $B$ is a complete normed algebra then $B$ is spectrally admissible. Indeed, $\sigma_{s u}^{B}(b) \cap \mathbb{D}_{\|b\|} \neq \emptyset$, for every $b \in B$.

Proof. If $B$ is complete then, from Lemma 3.4, we have $\sigma_{s u}^{B}(b)$ is non-empty (in fact, $\sigma_{s u}^{B}(b)$ contains the boundary of $\sigma^{L(B)}\left(L_{b}\right)$ and $\left.\sigma^{L(B)}\left(R_{b}\right)\right)$. Moreover, by Proposition 2.5 we have that $\sigma_{s u}^{B}(b) \subseteq \mathbb{D}_{\|b\|}$ and therefore, $\sigma_{s u}^{B}(b) \cap \mathbb{D}_{\|b\|}=\sigma_{s u}^{B}(b) \neq \emptyset$.

Next, we show that associative normed algebras are spectrally admissible, even when they are not complete. Later we shall see that this does not hold for non-associative algebras in general.

Proposition 4.5. Associative normed algebras with a unit are spectrally admissible.

Proof. Let $B$ be a normed associative algebra with a unit. If $B$ is complete then $B$ is spectrally admissible as showed in Proposition 4.4. Suppose now that $B$ is not complete, and let $\widehat{B}$ denotes the completion of $B$. Note that $\widehat{B}$ is a Banach algebra with a unit, so that the set of invertible elements of $\widehat{B}$ is open [5, Theorem 1.2.11]. We claim that $\sigma_{s u}^{\widehat{B}}\left(L_{b}\right) \subseteq \sigma_{s u}^{B}\left(L_{b}\right)$. Indeed, if $L_{b}-\lambda I: \widehat{B} \rightarrow \widehat{B}$ is not surjective then, from the associativity of $B$, it follows that $\left(L_{b}-\lambda I\right)(\widehat{B})$ does not contain any invertible element of $\widehat{B}$, so that the range of $L_{b}-\lambda I: \widehat{B} \rightarrow \widehat{B}$ is not dense in $\widehat{B}$ and hence $L_{b}-\lambda I: B \rightarrow B$ is not surjective. This proves that $\sigma_{s u}^{\widehat{B}}\left(L_{b}\right) \subseteq \sigma_{\text {su }}^{B}\left(L_{b}\right)$ as claimed. Therefore, from the fact that $\sigma^{L(\widehat{B})}\left(L_{b}\right) \subseteq \mathbb{D}_{\|b\| \|}$, it follows that $B$ is spectrally admissible because $\sigma_{s u}^{\widehat{B}}\left(L_{b}\right)$ is a non-empty set contained into $\sigma_{s u}^{B}(b) \cap \mathbb{D}_{\|b\|}$ (note that $L_{b}$ can be replaced by $R_{b}$ ).

In the setting of Problem 4.2, a sufficient condition for the automatic continuity of a dense range homomorphism $\theta: A \rightarrow B$ is that $\theta$ has a spectrally admissible range, as we establish in the next result.

Theorem 4.6. Let $A$ and $B$ be complete normed algebras with a unit, and let $\theta: A \rightarrow B$ be a dense range homomorphism. If $B$ is simple and if the range of $\theta$ is spectrally admissible, then $\theta$ is continuous.

Proof. Let $a \in A$. If the operator $L_{a}-\lambda I: A \rightarrow A$ is surjective then, from the surjectivity of $\theta: A \rightarrow \theta(A)$, we have that $\theta\left(L_{a}^{A}-\lambda I\right): A \rightarrow \theta(A)$ is surjective. But $\theta\left(L_{a}^{A}-\lambda I\right)=\left(L_{\theta(a)}-\lambda I\right) \theta$ so that $L_{\theta(a)}-\lambda I: \theta(A) \rightarrow \theta(A)$ is surjective. Consequently,

$$
\sigma_{s u}^{\theta(A)}\left(L_{\theta(a)}\right) \subseteq \sigma_{s u}^{A}\left(L_{a}\right)
$$

Similarly $\sigma_{s u}^{\theta(A)}\left(R_{\theta(a)}\right) \subseteq \sigma_{s u}^{A}\left(R_{a}\right)$. Let $e_{A}$ and $e_{B}$ denotes the unit of $A$ and $B$ respectively. Since $\theta\left(e_{A}\right)$ is a unit for $\theta(A)$, and $\theta(A)$ is dense in $B$, it follows that $\theta\left(e_{A}\right)=e_{B}$ so that $\theta$ is unital. Therefore $I-L_{\theta(a)}=L_{\theta\left(e_{A}-a\right)}=L_{e_{B}-\theta(a)}$. By hypothesis there exists $M>0$ such that, for every $a \in A$, the set

$$
\sigma_{s u}^{B}\left(e_{B}-\theta(a)\right) \cap M \mathbb{D}_{\left\|e_{B}-\theta(a)\right\|}=\left[\sigma_{s u}^{\theta(A)}\left(I-L_{\theta(a)}\right) \cup \sigma_{s u}^{\theta(A)}\left(I-R_{\theta(a)}\right)\right] \cap M D_{\left\|e_{B}-\theta(a)\right\|}
$$

is non-empty. Fix $a \in A$, and take

$$
\lambda \in\left[\sigma_{s u}^{\theta(A)}\left(I-L_{\theta(a)}\right) \cup \sigma_{s u}^{\theta(A)}\left(I-R_{\theta(a)}\right)\right] \cap M \mathbb{D}_{\left\|e_{B}-\theta(a)\right\|} .
$$

Thus, $|\lambda| \leq M\left\|e_{B}-\theta(a)\right\|$ and $\lambda \in \sigma_{s u}^{\theta(A)}\left(I-T_{\theta(a)}\right)$, where $T_{\theta(a)}$ denotes either $L_{\theta(a)}$ or $R_{\theta(a)}$. Obviously we have that $1-\lambda \in \sigma_{s u}^{\theta(A)}\left(T_{\theta(a)}\right)$ and $\sigma_{s u}^{\theta(A)}\left(T_{\theta(a)}\right) \subseteq \sigma_{s u}^{A}\left(T_{a}\right)$, by (4). Thus, we obtain $1-\lambda \in \sigma_{s u}^{A}\left(T_{a}\right)$ and, from the completeness of $A$, it follows that $|1-\lambda|<\|a\|$. Consequently, $1 \leq|\lambda|+|1-\lambda| \leq M\|e-\theta(a)\|+\|a\|$, which proves that $e$ is not in $\mathcal{S}(\theta)$, the separating subspace of $\theta$. Since $\mathcal{S}(\theta)$ is an ideal of $B$, and $B$ is simple, it follows that a $\mathcal{S}(\theta)=\{0\}$ and therefore $\theta$ is continuous from the closed graph theorem.

The next result is useful to prove the discontinuity of a dense range homomorphism. 
Proposition 4.7. Let $A$ and $B$ be complete normed algebras and $\theta: A \rightarrow B$ a dense range homomorphism. If the kernel of $\theta$ is closed then $\sigma_{\text {su }}^{\theta(A)}(\theta(a)) \neq \emptyset$, for every $a \in A$.

Proof. Let $\widehat{\theta}: A / \operatorname{ker} \theta \rightarrow \theta(A)$ be the homomorphism given by $\widehat{\theta}(a+\operatorname{ker} \theta)=\theta(a)$. If $\operatorname{ker} \theta$ is closed then $\widehat{\theta}$ is bijective and such that

$$
L_{\theta(a)}^{\theta(A)}=\widehat{\theta} L_{a+\operatorname{ker} \theta}^{A / \operatorname{ker} \theta} \widehat{\theta}^{-1} .
$$

It follows that $\sigma_{s u}^{\theta(A)}\left(L_{\theta(a)}\right)=\sigma_{s u}^{A / \operatorname{ker} \theta}\left(L_{a+\operatorname{ker} \theta}\right)$. Moreover, $\sigma_{s u}^{A / \operatorname{ker} \theta}\left(L_{a+\operatorname{ker} \theta}\right) \neq \emptyset$, because $A / \operatorname{ker} \theta$ is a complete normed algebra (see Lemma 3.4). Therefore,

$$
\sigma_{s u}^{\theta(A)}\left(L_{\theta(a)}\right)=\sigma_{s u}^{A / \operatorname{ker} \theta}\left(L_{a+\operatorname{ker} \theta}\right) \neq \emptyset,
$$

(and similarly $\sigma_{s u}^{\theta(A)}\left(R_{\theta(a)}\right)=\sigma_{s u}^{A / \operatorname{ker} \theta}\left(R_{a+\operatorname{ker} \theta}\right) \neq \emptyset$ ). Consequently $\sigma_{s u}^{\theta(A)}(\theta(a)) \neq \emptyset$, for every $a \in A$.

As we said above, the fact that the associativity is a superfluous hypothesis for the classical Rickart's theorem that we are considering is equivalent to answering Problem 4.2 affirmatively. From the above proposition we obtain that if $\theta(A)$ contains some element with empty surjective m-spectrum then, $\theta$ is automatically discontinuous.

Next, we provide an example of a complete normed algebra $B$, simple with a unit, that contains a dense subalgebra $B_{0}$ which have elements with empty surjective m-spectrum. Therefore, if $\theta: A \rightarrow B$ is a dense range homomorphism from a complete normed unital algebra onto $B_{0}$ then $\theta$ is discontinuous.

Example 4.8. Let $\mathcal{B}_{0}$ be a linear space generated by the set $\left\{e, e_{1}, e_{2}, \ldots\right\}$ and whose product is defined as follows

\begin{tabular}{r||ccccccccc} 
& $e$ & $e_{1}$ & $e_{2}$ & $e_{3}$ & $e_{4}$ & $e_{5}$ & $\ldots$ & $e_{k}$ & $\ldots$ \\
\hline \hline$e$ & $e$ & $e_{1}$ & $e_{2}$ & $e_{3}$ & $e_{4}$ & $e_{5}$ & & $e_{k}$ & \\
$e_{1}$ & $e_{1}$ & 0 & $e$ & $e_{1}$ & $e_{2}$ & $e_{3}$ & & $e_{k-2}$ & \\
$e_{2}$ & $e_{2}$ & 0 & 0 & $e$ & $e_{1}$ & $e_{2}$ & & $e_{k-3}$ & \\
$e_{3}$ & $e_{3}$ & 0 & $e_{1}$ & 0 & $e$ & $e_{1}$ & & $e_{k-4}$ & \\
$e_{4}$ & $e_{4}$ & 0 & $e_{3}$ & 0 & 0 & $e$ & & $e_{k-5}$ & \\
$e_{5}$ & $e_{5}$ & 0 & $e_{4}$ & 0 & 0 & 0 & & $e_{k-6}$ & \\
$e_{6}$ & $e_{6}$ & 0 & $e_{5}$ & 0 & 0 & 0 & & $e_{k-7}$ & \\
$e_{7}$ & $e_{7}$ & 0 & $e_{6}$ & 0 & 0 & 0 & & $e_{k-8}$ & \\
$e_{8}$ & $e_{8}$ & 0 & $e_{7}$ & 0 & 0 & 0 & & $e_{k-9}$ & \\
$\vdots$ & & & & & & & & $\vdots$ & \\
$e_{k-1}$ & $e_{k-1}$ & 0 & $e_{k-2}$ & 0 & 0 & 0 & & $e$ & \\
$e_{k}$ & $e_{k}$ & 0 & $e_{k-1}$ & 0 & 0 & 0 & & 0 & \\
$e_{k+1}$ & $e_{k+1}$ & 0 & $e_{k}$ & 0 & 0 & 0 & & 0 & \\
$\vdots$ & & & & & & & & &
\end{tabular}

We claim that $\sigma_{s u}^{B_{0}}\left(e_{2}\right)=\emptyset$. To prove the claim note that $L_{e_{2}}$ is surjective because $L_{e_{2}}\left(e_{3}\right)=e$ and $L_{e_{2}}\left(e_{2+k}\right)=e_{k-1}$, for every $k \geq 2$. On the other hand, if $\lambda \neq 0$ then $L_{e_{2}}-\lambda I$ is surjective because, from the equalities

$$
\begin{aligned}
\left(e_{2}-\lambda e\right)\left(e_{2}+\lambda e\right) & =-\lambda^{2} e, \\
\left(e_{2}-\lambda e\right) e_{1} & =-\lambda e_{1} \\
\left(e_{2}-\lambda e\right) e_{2} & =-\lambda e_{2} \\
\left(e_{2}-\lambda e\right) e_{3} & =e-\lambda e_{3}, \\
\left(e_{2}-\lambda e\right) e_{k+2} & =e_{k-1}-\lambda e_{k+2}, \text { for } k>1,
\end{aligned}
$$

it follows that the range of $\left(L_{e_{2}}-\lambda I\right)$ contains set $\left\{e_{,} e_{1}, e_{2}, \ldots\right\}$ and, therefore, also contains the algebra $B_{0}$. Thus $L_{e_{2}}-\lambda I$ is surjective. Similarly $R_{e_{2}}-\lambda I$ is surjective for every $\lambda \in \mathbb{C}$, and hence $\sigma_{s u}^{B_{0}}\left(e_{2}\right)=\emptyset$. Let $B$ be the the linear 
space of all elements $\sum_{i=0}^{\infty} \alpha_{i} e_{i}$ such that $\sum_{i=0}^{\infty}\left|\alpha_{i}\right|<\infty$, where $e_{0}:=$ e. By defining $\left\|\sum_{i=0}^{\infty} \alpha_{i} e_{i}\right\|:=\sum_{i=0}^{\infty}\left|\alpha_{i}\right|$ we obtain a complete norm on B. Moreover, since $\left\|e_{j} \sum_{i=0}^{\infty} \beta_{i} e_{i}\right\| \leq \sum_{k=1}^{\infty}\left|\beta_{k}\right|$, it follows that $\left\|\left(\sum_{i=0}^{\infty} \alpha_{i} e_{i}\right)\left(\sum_{i=0}^{\infty} \beta_{i} e_{i}\right)\right\| \leq \sum_{i=0}^{\infty}\left|\alpha_{i}\right| \sum_{i=0}^{\infty}\left|\beta_{i}\right|$, so that B is nothing but the completion of $B_{0}$. Obviously e is a unit of $B$. We claim now that $B$ is simple. Indeed, if $\sum_{i=0}^{\infty} \alpha_{i} e_{i}$ is such that $\alpha_{m} \neq 0$ for some $m \geq 3$ then, $\left(\sum_{i=0}^{\infty} \alpha_{i} e_{i}\right) e_{m+1}=\alpha_{0} e_{m+1}+\alpha_{1} e_{m-1}+\alpha_{2} e_{m-2}+\ldots+\alpha_{m-1} e_{1}+\alpha_{m} e_{0}$, so that

$$
e_{m+1}\left[\left(\sum_{i=0}^{\infty} \alpha_{i} e_{i}\right) e_{m+1}\right]=\alpha_{m-2} e_{m}+\alpha_{m} e_{m+1} .
$$

Consequently,

$$
e_{m}\left(e_{m+1}\left[\left(\sum_{i=0}^{\infty} \alpha_{i} e_{i}\right) e_{m+1}\right]\right)=e_{m}\left(\alpha_{m-2} e_{m}+\alpha_{m} e_{m+1}\right)=\alpha_{m} e .
$$

This proves that the ideal of $B$ generated by $\sum_{i=0}^{\infty} \alpha_{i} e_{i}$ contains the unit of $B$. On the other hand, if $\sum_{i=0}^{\infty} \alpha_{i} e_{i}$ is such that $\alpha_{m}=0$ for every $m \geq 3$ then, $\sum_{i=0}^{\infty} \alpha_{i} e_{i}=\alpha_{0} e+\alpha_{1} e_{1}+\alpha_{2} e_{2}$.

In this case, if $\alpha_{0} \neq 0$ then, $\left(\left(\alpha_{0} e+\alpha_{1} e_{1}+\alpha_{2} e_{2}\right) e_{1}\right) e_{2}=\alpha_{0} e_{1} e_{2}=\alpha_{0} e$, while if $\alpha_{1} \neq 0$ then,

$$
\left[\left(\left(\alpha_{0} e+\alpha_{1} e_{1}+\alpha_{2} e_{2}\right) e_{2}\right) e_{1}\right] e_{2}=\alpha_{1} e,
$$

and, finally, if $\alpha_{2} \neq 0$ then, $\left[\left(\left(\alpha_{0} e+\alpha_{1} e_{1}+\alpha_{2} e_{2}\right) e_{3}\right) e_{1}\right] e_{2}=\alpha_{2} e$. This proves that $B$ is simple.

\section{Final remarks: Potential application areas of this approach}

From the thirties, non-associative algebras have been used in genetics to describe hereditary processes, in order to determine which genetic characters are more stable for certain reproductive processes. As shown in $[1,2,7-10,12,21,28]$, hereditary stability problems are strongly linked with spectral theory. Because of this, several techniques have been designed to obtain results in this framework by avoiding the problem of the lack of a non-associative spectral theory, as well as the generalized use of infinite-dimensional algebras. In line with this arguments is the following sentence of M. L. Reed [21]:

We conclude that this area of research activity is full of possibilities for the future, not only for mathematicians, but also for geneticists seeking a more systematic and powerful way to model real genetics. Non-associative algebra, in general, is currently a very active field of mathematical research. However, in comparison with the body of literature of other classes of non-associative algebras (e.g., Lie algebras or Jordan algebras), the study of the algebras associated with the problems of genetic inheritance is still in its infancy... For whatever reason, these "genetic algebras" are not widely discussed or studied presently by American mathematicians. Hopefully, this article will open an avenue for future discussion and research into this fascinating class of non-associative algebras and their relationship to the science of genetic inheritance.

We fully endorse the idea that this is a subject with plenty of future, and it seems that establishing a concept of spectrum like the one provided in this paper is a good starting point to develop a spectral theory which may be useful to deal with problems in these areas.

In this section we shall not develop the required framework to consider genetic algebras in a deeper way (a work addressed specifically to this question is [18]). In what follows, we simply provide a flavour of how to apply some of the above results in the framework of evolution algebras. 
We recall from $[13,23,26]$ that an evolution algebra is nothing but the space $l_{1}$ provided with multiplication table such that $e_{i} e_{j}=0$, if $i \neq j$, while $e_{i}^{2}=\sum_{j \in \mathbb{N}} \gamma_{i j} e_{j}$ with $\sum_{j=1}^{\infty}\left|\gamma_{i j}\right|=1$, for every $i \in \mathbb{N}$ (usually the constants $\gamma_{i j}$ are named structure coefficients). In the algebraic formulation of non-Mendelian Genetics, $e_{i}$ might denote the genotypes of organelle population, and the coefficients $\gamma_{i j}$ can be interpreted as the rate of genotype $e_{j}$ produced by genotype $e_{i}$.

Consider an evolution algebra $B$ such that $\gamma_{i j}=0$ if $i<j$ and $\gamma_{i i} \neq 0$, for every $i \in \mathbb{N}$. The first condition means that it is not likely that the genotype $e_{j}$ be obtained by self-replication of $e_{i}$ whenever $j<i$ and, similarly, the assumption $\gamma_{i i} \neq 0$, for every $i \in \mathbb{N}$, means that the probability that $e_{i}$ be obtained from self-replication of $e_{i}$ is non-zero.

Note that $B$ is m-semisimple because $\{b \in B: \rho(b)=0\}=\{0\}$. Indeed, if $b \in B \backslash\{0\}$ is given by $b=\sum_{k \in \mathbb{N}} \beta_{k} e_{k}$, and if $k_{0}:=\min \left\{k \in \mathbb{N}: \beta_{k} \neq 0\right\}$, then $\lambda_{0}=\beta_{k_{0}} \gamma_{k_{0} k_{0}}$ belongs to $\sigma_{m}^{B}(b)$, because $e_{k_{0}}$ is not in the range of $L_{b}-\lambda_{0} I$. Consequently, from Theorem 3.5 and Corollary 3.6, we obtain the next result for these algebras of the non-Mendelian genetics.

Theorem 5.1. Every homomorphism from a complete normed algebra $A$ onto an evolution algebra $B$ is continuous whenever the structure coefficients $\gamma_{i j}$ of the algebra $B$ are such that $\gamma_{i j}=0$ if $i<j$ and $\gamma_{i i} \neq 0$, for every $i \in \mathbb{N}$. In particular, such evolution algebras B have uniqueness of the complete norm topology.

Biologically, as pointed out already, the structure coefficients $\gamma_{i i}$ that are mentioned in the above theorem denote the rate of the genotype (or organelle) $e_{i}$ produced by itself.

\section{References}

[1] V. M. Abraham, Linearizing Quadratic Transformations in Genetic Algebras, Proc. London Math. Soc. (3) 40 (1980) $346-363$.

[2] V. M. Abraham, The induced Linear Transformation in a Genetic Algebra, Proc. London Math. Soc. (3) 40 (1980) 364-384.

[3] A. A. Albert, Non-Associative Algebras: I. Fundamental Concepts and Isotopy, Ann. Math. 43 (1942) 685-707.

[4] R. E. Beck and B. Kolman (Eds), Computers in Nonassociative Rings and Algebras, New York, Academic Press (1977).

[5] F. F. Bonsall and J. Duncan, Complete Normed Algebras, Springer (1973).

[6] H. G. Dales, Banach Algebras and Automatic Continuity, London Math. Soc. Monographs 24, Clarendon Press, Oxford (2000).

[7] I. M. H. Etherington, Genetic Algebras, Proc. Roy. Soc. Edinburgh 59 (1939), 242-258.

[8] I. M. H. Etherington, Special Train Algebras, Quart. J. of Math. Oxford (2) 12 (1941) 1-8

[9] H. Gonshor, Special Train Algebras Arising in Genetics, Proc. Edin. Math. Soc. (2) 12 (1960) 41-53.

[10] H. Gonshor, Contributions to Genetic Algebras, Proc. Edinburgh Math. Soc. 17 (1971) 289-298.

[11] Y. Gulak, Algebraic Properties of Some Quadratic Dynamical Systems, Adv. Appl. Math. 35 (2005) 407-432.

[12] P. Holgate, Sequences of Powers in Genetic Algebras, J. London Math. Soc. 42 (1967) 489-496.

[13] P. Holgate, Population Algebras, J. R. Statist. Soc. 43 (1981) 1-19.

[14] B. E. Johnson, The Uniqueness of the (Complete) Norm Topology, Bull. Amer. Math. Soc. 73 (1967) 537-539.

[15] K. B. Laursen and M. M. Neumann, An Introduction to Local Spectral Theory, Clarendon Press, Oxford series (2000).

[16] J. Lohmus, E. Paal and L. Sorgsepp, About Nonassociativity in Mathematics and Physics, Acta Applicandae Mathematicae 50 (1998) 3-31.

[17] J. C. Marcos and M. V. Velasco, The Jacobson Radical of an Non-Associative Algebra and the Uniqueness of the Complete Norm Topology, Bull.London Math. Soc. 42 (2010) 1010-1020.

[18] J. C. Marcos and M. V. Velasco, Non-Associative Algebras in Genetics, work in progress.

[19] S. Okubo, Introduction to Octonion and Other Non-Associative Algebras in Physics, Cambridge Univ. Press (1995).

[20] T. W. Palmer, Banach Algebras and the General Theory of *-algebras, vol. I, Cambridge University Press (1994).

[21] M. L. Reed, Algebraic Structure of Genetic Inheritance, Bull. Amer. Math. Soc. 34 (1997) 107-130.

[22] C. E. Rickart, The General Theory of Banach Algebras, Van Nostrand (1960).

[23] G. A. Ringwood, The Structure of Poisson Algebras, IMA J. Maths Applied in Medicine and Biology 2 (1985) 69-73.

[24] Schafer, Richard D., An Introduction to Nonassociative Algebras, Dover Publications (1955).

[25] A. Rodríguez-Palacios and M. V.Velasco, A Non-Associative Rickart's Dense-Range-Homomorphism Theorem, Quart. J. of Math., Oxford 54 (2003) 367-376.

[26] J. P. Tian, Evolution Algebras and Their Applications, Lecture Notes in Mathematics vol. 1921, Springer-Verlag (2008).

[27] M. V. Velasco, Spectral Theory for Non-Associative Complete Normed Algebras and Automatic Continuity, J. Math. Anal. Appl. 351 (2009) 97-106.

[28] A. Wörz-Busekros, Algebras in Genetics, Lecture Notes in Biomathematics, vol. 36, Springer-Verlag (1980). 